\title{
circRNA Hsa_circ_0020850 Silence Represses the Development of Lung Adenocarcinoma via Regulating miR-195-5p/IRS2 Axis
}

This article was published in the following Dove Press journal: Cancer Management and Research

\author{
Tuye Xin' \\ Shuangshuang $\mathrm{Li}^{\prime}$ \\ Ying Zhang' \\ Xiayizha Kamali ${ }^{\prime}$ \\ Hui Liu' \\ Tengfei Jia ${ }^{2}$ \\ 'Department of Respiration, The First \\ Affiliated Hospital of Xinjiang Medical \\ University, Urumqi, Xinjiang 830054, \\ People's Republic of China; ${ }^{2}$ Department \\ of Gastrointestinal Cancer Surgery, The \\ First Affiliated Hospital of Xinjiang \\ Medical University, Urumqi, Xinjiang, \\ 830054, People's Republic of China
}

Background: The dysregulated circular RNAs (circRNAs) are relevant to lung adenocarcinoma development. Nevertheless, the function and mechanism of hsa_circ_0020850 (circ_0020850) in lung adenocarcinoma development are uncertain.

Methods: A total of 35 lung adenocarcinoma patients were recruited, and the tumor and normal tissue samples were harvested. A549 and PC-9 cells were exhibited for the experiments in vitro. circ_0020850, microRNA-195-5p (miR-195-5p) and insulin receptor substrate 2 (IRS2) abundances were detected via quantitative reverse transcription-polymerase chain reaction or Western blot. Cell proliferation, apoptosis, migration and invasion were measured via cell counting kit-8 (CCK8) assay, colony formation, flow cytometry, transwell and Western blot. The relationship between miR-195-5p and circ_0020850 or IRS2 was tested via dual-luciferase reporter analysis. The function of circ_0020850 on cell growth in vivo was measured via xenograft model.

Results: circ_0020850 expression was enhanced in lung adenocarcinoma tissues and cells. circ_0020850 silence suppressed cell proliferation, migration and invasion and facilitated apoptosis. miR-195-5p was targeted via circ_0020850, and its knockdown reversed the inhibitive effect of circ_0020850 silence on lung adenocarcinoma development. IRS2 was targeted via miR-195-5p, and miR-195-5p inhibited cell proliferation, migration and invasion and induced apoptosis via decreasing IRS2. circ_0020850 knockdown decreased IRS2 expression via regulating miR-195-5p. circ_0020850 down-regulation decreased lung adenocarcinoma xenograft tumor growth.

Conclusion: circ_0020850 knockdown repressed lung adenocarcinoma cell proliferation, migration and invasion and promoted apoptosis via regulating miR-195-5p and IRS2.

Keywords: lung adenocarcinoma, circ 0020850, IRS2, miR-195-5p

\section{Introduction}

Lung cancer is one common cancer with high mortality and is classified as smallcell and non-small-cell lung cancer. ${ }^{1}$ Lung adenocarcinoma is the predominant histological subtypes of non-small-cell lung cancer. ${ }^{2}$ In spite of the lots of improvements in understanding the pathogenesis of lung adenocarcinoma, the outcomes of patients remain poor. ${ }^{2}$ Therefore, much hope is placed in exploring new target for the treatment of lung adenocarcinoma.

Circular RNAs (circRNAs) are the covalently closed biomolecules and exhibit key functions via serving as microRNA (miRNA) sponges. ${ }^{3}$ Lots of circRNAs are aberrantly expressed and might play important roles in lung adenocarcinoma. ${ }^{4}$ For
Correspondence: Tengfei lia

Department of Gastrointestinal Cancer Surgery, The First Affiliated Hospital of

Xinjiang Medical University, Xinshi District,

Urumqi, Xinjiang 830054, People's Republic

of China

Tel +86-I59-8I77I752

Email xI5899266774@I63.com 
example, hsa_circ_0001946 could promote cell growth via regulating miR-135a-5p and Sirtuin 1 in lung adenocarcinoma. ${ }^{5}$ Moreover, circRNA sex-determining region Y-related high-mobility group box 4 (SOX4) knockdown represses lung adenocarcinoma cell proliferation, migration and invasion via modulating miR-1270 and pleiomorphic adenoma gene-like $2 .^{6}$ The circRNA hsa_circ_0020850 (circ_0020850), derived from nucleoporin 98 and 96 precursor (NUP98), is one abnormally expressed circRNA analyzed via GSE101684 microarray data on the basis of report of Zhao et al. ${ }^{7}$ Nevertheless, the function and regulatory mechanism of circ_0020850 in lung adenocarcinoma development are uncertain.

miRNAs could regulate gene expression through translational inhibition or degradation, which are relevant to lung cancer development. ${ }^{8}$ The previous study reports miR-195-5p as a cancer-related miRNA in lung adenocarcinoma. ${ }^{9}$ Moreover, miR-195-5p could be used as an important marker for the detection of lung cancer. ${ }^{10}$ The increasing evidences suggest that miR-195-5p could repress lung adenocarcinoma development via regulating apelin and $\mathrm{T}$ cell activation. ${ }^{11,12}$ However, whether miR$195-5 p$ is relevant to the regulation of circ_0020850 on lung adenocarcinoma development is unclear. Insulin receptor substrate 2 (IRS2) is implicated in the reduced survival of lung adenocarcinoma patients. ${ }^{13}$ Furthermore, IRS2 contributes to lung cancer cell proliferation, migration and invasion. ${ }^{14,15}$ Nevertheless, little is known about if IRS2 takes part in the regulation of circ_0020850 on lung adenocarcinoma development.

In this research, we measured circ_0020850 in lung adenocarcinoma and assessed its function on cancer development in vitro and in vivo. Moreover, we explored the interaction network of circ_0020850/miR-195-5p/IRS2.

\section{Materials and Methods}

\section{Patient Tissues}

The tumor tissues and normal samples were harvested from 35 lung adenocarcinoma patients untreated via other therapy before resection from The First Affiliated Hospital of Xinjiang Medical University. The samples were stored at $-80^{\circ} \mathrm{C}$. The written informed consent was provided via every subject. This research was permitted via the ethics committee of The First Affiliated Hospital of Xinjiang Medical University and based on the principles of the Helsinki Declaration.

\section{Cell Culture}

Human lung adenocarcinoma cell lines A549 (Procell, Wuhan, China) and PC-9 (Beijing Future Biotech, Beijing, China) cells grew in RPMI-1640 medium (Thermo Fisher, Waltham, MA, USA) plus 10\% fetal bovine serum (Gibco, Gran Island, NY, USA) and 1\% penicillin/streptomycin (Transgen, Beijing, China) in 5\% $\mathrm{CO}_{2}$ at $37^{\circ} \mathrm{C}$. Human bronchial cell line BEAS-2B cells (Procell) grew in the specific BEGM medium (Procell) in $5 \% \mathrm{CO}_{2}$ at $37^{\circ} \mathrm{C}$.

\section{Quantitative Reverse Transcription Polymerase Chain Reaction (qRT-PCR)}

The RNA was extracted after treatment of Trizol reagent (Thermo Fisher) following the instructions as previously reported. ${ }^{16}$ The RNA was applied to cDNA generation through the specific reverse transcription kit (Fulengen, Guangzhou, China). Subsequently, the cDNA was used for qRT-PCR via mixing with SYBR (Toyobo, Tokyo, Japan) as well as specific primers (Genscript, Nanjing, China). The primers included circ_0020850 (sense, 5'TAAAGATCGCCTGGCTCAGT-3'; antisense, 5'GGTTGTAGCCTGGCCAAAT-3'), IRS2 (sense, 5'TCTCAGGAAAAGCAGCGAGG-3'; antisense, 5'-TCAC GTCGATGGCGATGTAG-3'), miR-195-5p (sense, 5'ACACTCCAGCTGGGTAGCAGCACAGAAAT-3'; antisense, 5'-TGGTGTCGTGGAGTCG-3'), U6 (sense, 5'AATTGGAACGATACAGAGAAGATTAGC-3'; antisense, 5'-TATGGAACGCTTCACGAATTTG-3'), and GAPDH (sense, 5'-GAATGGGCAGCCGTTAGGAA-3'; antisense, 5'-AAAAGCATCACCCGGAGGAG-3'). With $\mathrm{U} 6$ or GAPDH as an internal reference, the relative RNA expression was calculated via $2^{-\Delta \Delta \mathrm{Ct}}$ method. ${ }^{17}$

\section{Cell Transfection}

The IRS2 overexpression vector (pcDNA-IRS2) was constructed via cloning the full-length sequence of IRS2 into pcDNA3.1 vector (Thermo Fisher), with the empty vector as negative control (pcDNA-NC). To knock down circ_0020850, the siRNA for circ_0020850 (sicirc_0020850, 5'-CUGGCUCAGUCAGGUACAACU-3') was generated via Genechem (Shanghai, China), with siNC (5'-AACAGUCGCGUUUGCGACUGG-3') as the siRNA negative control. Moreover, miR-195-5p mimic (5'-UAGCAGCACAGAAAUAUUGGC-3'), negative control of mimic (miR-NC, 5'-CGAUCGCAUCAGCA UCGAUUGC-3'), miR-195-5p inhibitor (anti-miR-195- 
5p, 5'-GCCAAUAUUUCUGUGCUGCUA-3'), as well as negative control of inhibitor (anti-miR-NC, 5'CUAACGCAUGCACAGUCGUACG-3'), were generated via Genechem. A549 and PC-9 cells were transfected with the vectors or oligonucleotides via Lipofectamine 3000 (Thermo Fisher) for $24 \mathrm{~h}$.

\section{Cell Proliferation Analysis}

Cell proliferation was assessed via cell viability and colony formation. For cell viability analysis, $5 \times 10^{3}$ A549 and PC-9 cells were added into 96-well plates. After incubating for $0,24,48$ or $72 \mathrm{~h}, 10 \mu \mathrm{L}$ of cell counting kit-8 (CCK8) (Beyotime, Shanghai, China) was added to every well. Following culture for $3 \mathrm{~h}$, the optical density (OD) value was detected at $450 \mathrm{~nm}$ through a microplate reader (Molecular Devices, Sunnyvale, CA, USA).

For colony formation analysis, 500 A549 and PC-9 cells were placed into 6-well plates and maintained for 10 days. Next, the clones were fixed and dyed with $0.1 \%$ crystal violet (Beyotime). The formed clones were observed and counted with microscope (Nikon, Tokyo, Japan).

\section{Flow Cytometry}

Cell apoptotic rate was examined via flow cytometry using Annexin V-FITC apoptosis kit (Sigma, St. Louis, MO, USA). $2 \times 10^{5}$ A549 and PC-9 cells were placed in 6well plates and grown for $72 \mathrm{~h}$. Next, cells were incubated with Annexin V-FITC binding buffer, and dyed with Annexin V-FITC and PI, followed via examining using flow cytometer (Agilent, Hangzhou, China). Cell apoptotic rate was presented as the percentage of cells in with Annexin $\mathrm{V}-F I T C^{+}$and $\mathrm{PI}^{ \pm}$.

\section{Transwell Analysis}

The abilities of cell migration and invasion were detected via transwell analysis using transwell chamber (BD, Franklin Lakes, NJ, USA). For migration analysis, $1 \times$ $10^{5}$ A549 and PC-9 cells in medium without serum were placed in the top chambers, and $600 \mu \mathrm{L}$ of medium with $10 \%$ serum was injected in the lower chamber. Following $24 \mathrm{~h}$, cells were dyed with $0.1 \%$ crystal violet and then observed under a microscope (magnification $\times 100$ ) with 4 random fields. For invasion analysis, $5 \times 10^{5}$ A549 and PC-9 cells were added in the top chambers coated via Matrigel (BD), and the other procedures were similar to migration analysis.

\section{Western Blot}

The protein was isolated using RIPA buffer (Solarbio, Beijing, China), and then the concentration was detected with a BCA kit (Thermo Fisher). The protein samples were separated via SDS-PAGE and transferred to polyvinylidene difluoride membranes (Solarbio). After blocking in $5 \%$ fat-free milk, the membranes were interacted with primary and secondary antibodies (Abcam, Cambridge, MA, USA), including anti-CyclinD1 (ab134175, 1:10,000 dilution), anti-Bcl-2 (ab196495, 1:500 dilution), antiCleaved caspase-3 (Cleaved-casp-3) (ab2302, 1:200 dilution), anti-Vimentin (ab137321, 1:3000 dilution), anti-Ncadherin (ab76057, 1:1000 dilution), anti-E-cadherin (ab227639, 1:100 dilution), anti-IRS2 (ab134101, 1:1000 dilution), GAPDH (ab22555, 1:5000 dilution) and IgG conjugated via HRP (ab205718, 1:8000 dilution). GAPDH acted as a loading control. After exposing to ECL reagent (Thermo Fisher), the protein blots were visualized, and next tested via Image $\mathrm{J}$ software $(\mathrm{NIH}$, Bethesda, MD, USA).

\section{Dual-Luciferase Reporter Analysis}

The target correlation of miR-195-5p and circ_0020850 or IRS2 was predicted via starBase (http://starbase.sysu. edu.cn/). The luciferase reporter vectors (circ_0020850WT or IRS2 3'UTR-WT) were constructed via inserting the sequence of circ_0020850 or IRS2 containing miR$195-5$ p complementary sites in the pmirGlO vectors (Promega, Madison, WI, USA) via endonuclease sites Nhe I and Xba I. The luciferase reporter plasmids (circ_0020850-MUT or IRS2 3'UTR-MUT) were constructed via mutating the binding sequence. These constructed vectors and miR-195-5p mimic or miR-NC were transfected into A549 and PC-9 cells for $24 \mathrm{~h}$. The luciferase activity was measured through dual-luciferase analysis kit (Promega) and a GloMax 20/20 Luminometer (Promega).

\section{Xenograft Model}

The lentiviral vector of sh-circ_0020850 or sh-NC was generated via GeneChem and infected to PC-9 cells. The stably transfected cells were selected via puromycin. The 6-week-old BALB/c nude mice were provided via Charles River (Beijing, China), and arbitrarily divided into two groups ( $n=6 /$ group): sh-circ_0020850 or sh-NC group. In sh-circ_0020850 group, $5 \times 10^{6}$ PC-9 cells with stable transfection of sh-circ_0020850 were 
subcutaneously injected into nude mice. In sh-NC group, $5 \times 10^{6}$ PC-9 cells with stable transfection of sh-NC were subcutaneously inoculated into mice. After cell injection for 7 days, tumor size was monitored every 4 days, and volume was calculated via $1 / 2 \times$ length $\times$ width $^{2}$. After cell injection for 27 days, mice were euthanized via 5\% isoflurane. Tumor samples were weighed and harvested for detection of circ_0020850, miR-195-5p and IRS2 expression. The experiment has obtained the approval of the Animal Ethical Committee of The First Affiliated Hospital of Xinjiang Medical University and was conducted according to the National Institutes of Health.

\section{Statistical Analysis}

The experiments were conducted 3 times, unless otherwise indicated. The data were displayed as mean $\pm \mathrm{SD}$. The linear correlation of circ_0020850, miR-195-5p and IRS2 expression in lung adenocarcinoma was tested via Pearson correlation analysis. Statistical analysis was carried out using Student's $t$-test or ANOVA with Tukey's test via GraphPad Prism 7 (GraphPad Inc., La
Jolla, CA, USA). The difference between groups was significant at $P<0.05$.

\section{Results}

\section{circ_0020850 Expression is Enhanced in Lung Adenocarcinoma}

To analyze the circRNA involved in lung adenocarcinoma development, we searched and analyzed 20 common circRNAs (10 up-regulation and 10 down-regulation) using GSE101684 (Figure 1A). circ_0020850 is one circRNA with the most change in expression than others including circ_404458 and circ_406826 with similar expression in the heatmap (Supplementary Figure 1). Furthermore, we measured circ_0020850 expression in lung adenocarcinoma tissues and cells. As shown in Figure 1B, circ_0020850 level was evidently enhanced in tumor tissues compared with normal samples $(\mathrm{n}=35)$. Moreover, higher abundance of circ_0020850 was displayed in A549 and PC-9 cells than BEAS-2B cells (Figure 1C). These results showed that the increased circ_0020850 might be associated with lung adenocarcinoma development.
A

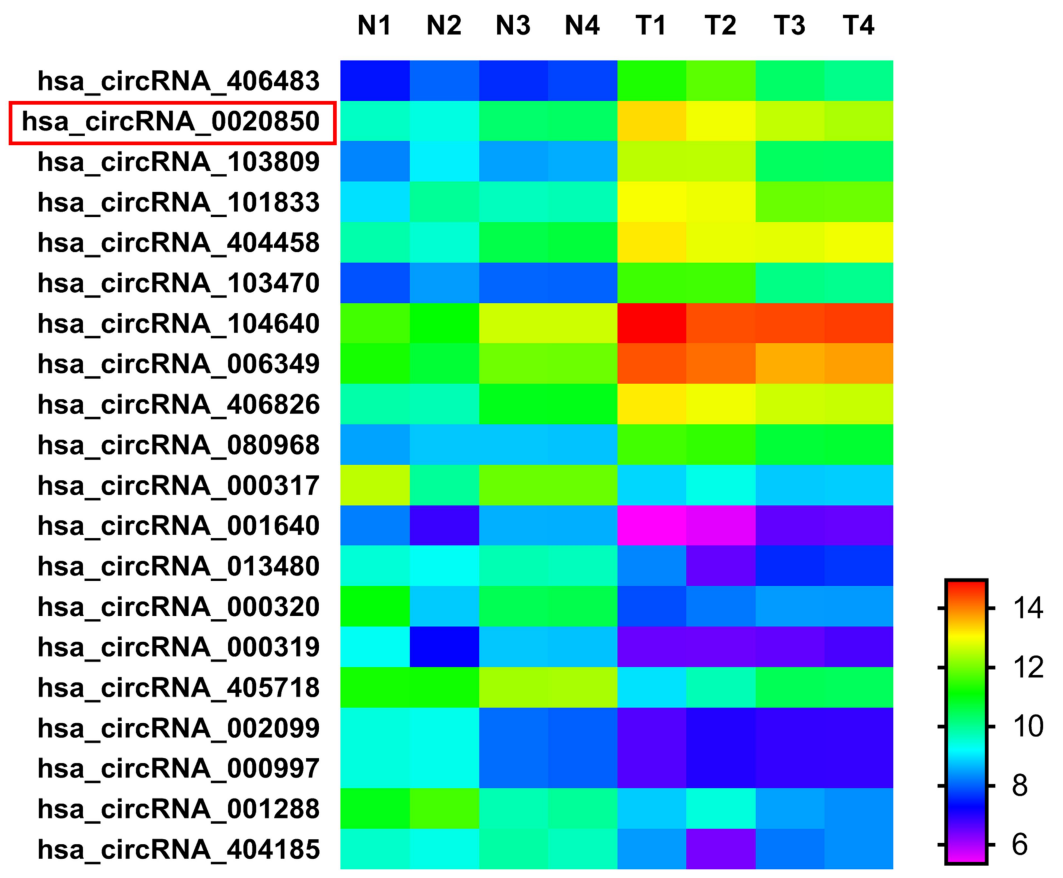

B

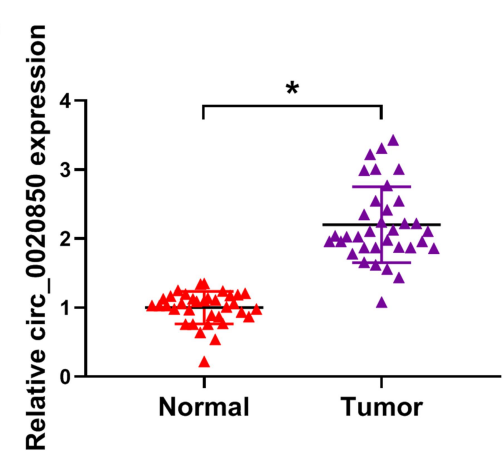

C

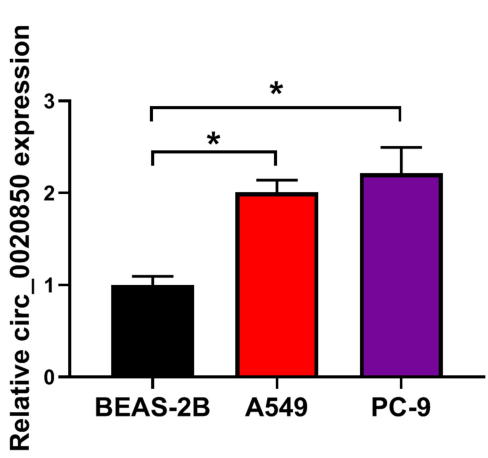

Figure I Circ_0020850 level in lung adenocarcinoma. (A) The common dysregulated circRNAs in lung adenocarcinoma predicted via GSEI0I684. (B) circ_0020850 level was detected in lung adenocarcinoma tissues and normal tissues. $n=35$. (C) circ_0020850 abundance was measured in A549, PC-9 and BEAS-2B cells. $*$ P $<0.05$. 
A

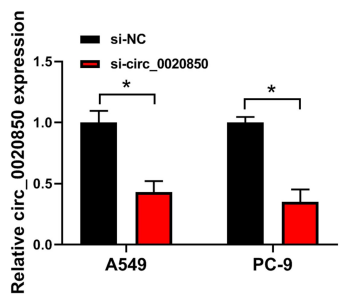

B

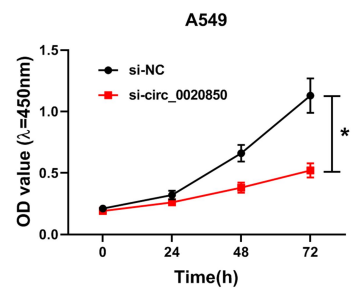

C

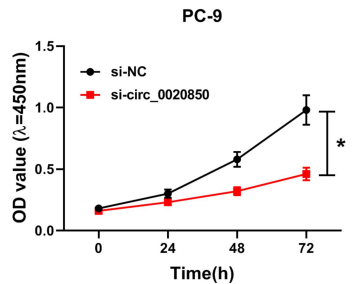

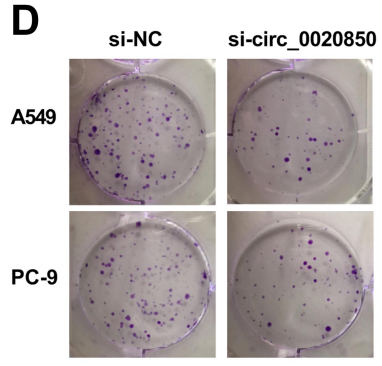

F

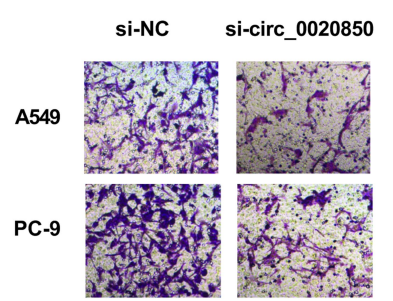

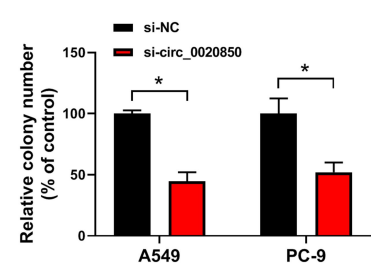

A54

E
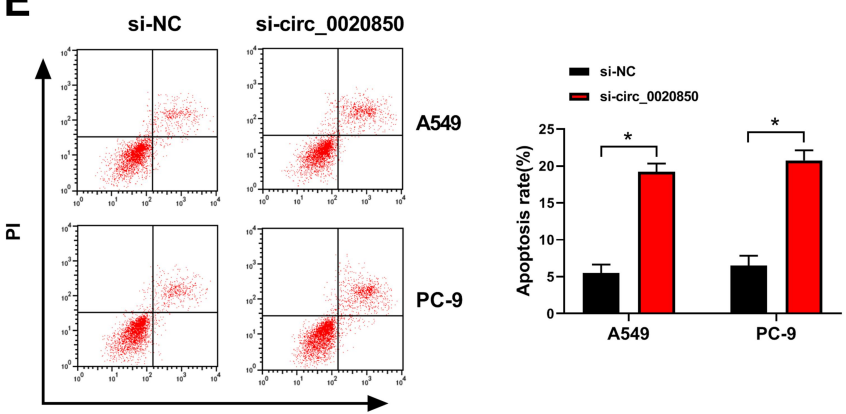

G
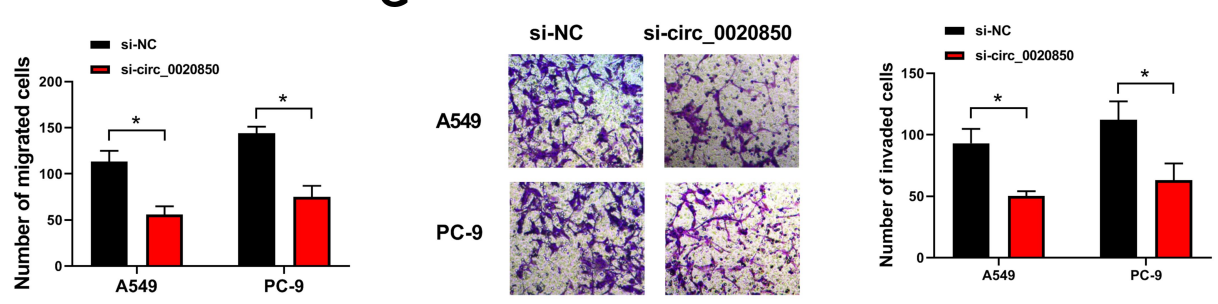

H
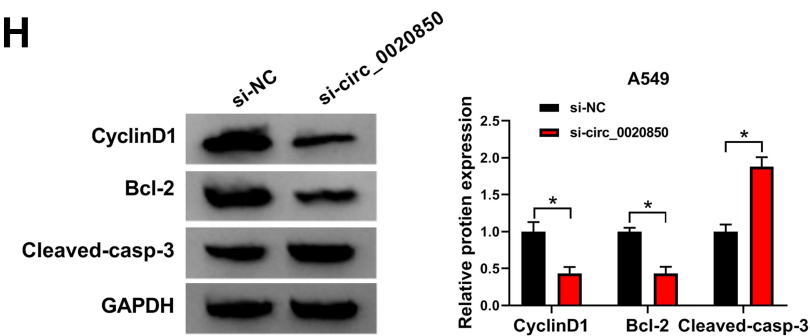

I
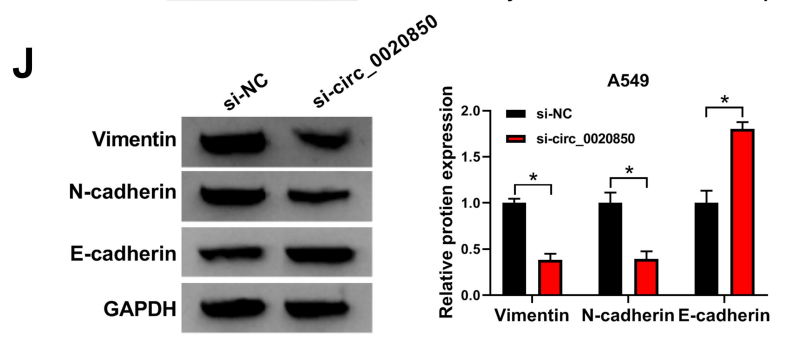
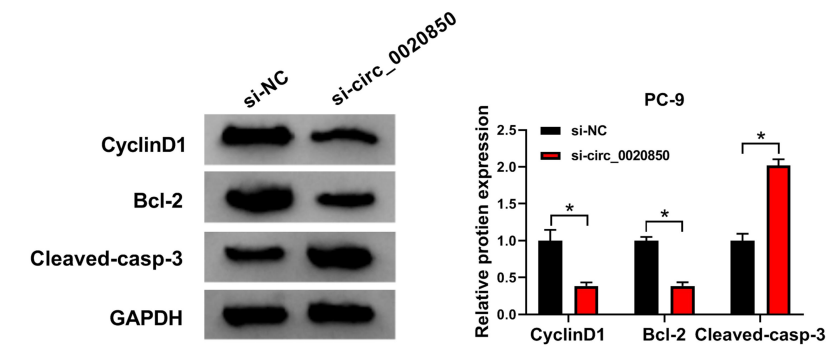

$\mathbf{K}$
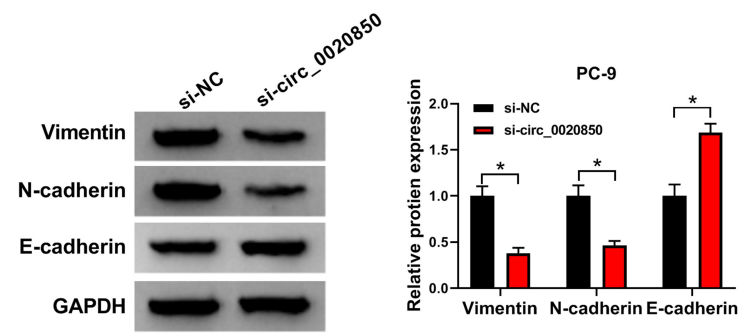

Figure 2 The influence of circ_0020850 on lung adenocarcinoma development. (A) circ_0020850 abundance was examined in cells with transfection of si-circ_0020850 or si-NC. (B and C) Cell viability was detected in cells with transfection of si-circ_0020850 or si-NC at 0, 24, 48 or 72 h. (D) The colony formation was analyzed in cells with transfection of si-circ_0020850 or si-NC. (E) Cell apoptotic rate was measured in cells with transfection of si-circ_0020850 or si-NC. (F and G) Cell migration and invasion were examined in cells with transfection of si-circ_0020850 or si-NC. (H-K) CyclinDI, Bcl-2, Cleaved-casp-3, Vimentin, N-cadherin and E-cadherin protein abundances were detected in cells with transfection of si-circ_0020850 or si-NC. $* P<0.05$.

\section{circ_0020850 Knockdown Inhibits Lung Adenocarcinoma Development}

To test the function of circ_0020850 on lung adenocarcinoma development, A549 and PC-9 cells were transfected with si-circ_0020850 or si-NC. As shown in Figure 2A, circ_0020850 abundance was effectively reduced by more than $60 \%$ via transfection of sicirc_0020850. circ_0020850 knockdown evidently 
reduced cell viability at $72 \mathrm{~h}$ (Figure $2 \mathrm{~B}$ and $\mathrm{C}$ ). In addition, circ_0020850 silence evidently inhibited the clone ability of A549 and PC-9 cells (Figure 2D). Furthermore, circ_0020850 interference promoted cell apoptosis (Figure 2E). Besides, the data of transwell analysis showed that circ_0020850 down-regulation markedly suppressed cell migration and invasion (Figure 2F and $\mathrm{G}$ ). Additionally, the related protein markers were detected. Results displayed that circ_0020850 knockdown evidently decreased the proproliferation CyclinD1 and anti-apoptotic Bcl-2 expression and elevated pro-apoptotic Cleaved-casp-3 level (Figure 2H and I). Moreover, circ_0020850 knockdown significantly reduced Vimentin and N-cadherin levels and increased E-cadherin expression in the two cells (Figure 2J and $\mathrm{K}$ ). These results indicated that circ_0020850 silence repressed lung adenocarcinoma development in vitro.

\section{miR-195-5p is Targeted via circ_0020850}

To explore the regulatory network of circ_0020850, the target of circ_0020850 was searched via starBase. The 8 predicted miRNAs were analyzed, and miR-195-5p was the only one up-regulated via circ_0020850 knockdown in A549 and PC-9 cells (Supplementary Figure 2). miR195-5p was a target and the target sequence of circ_0020850 and miR-195-5p is exhibited in Figure $3 \mathrm{~A}$. To confirm the target association, dual-luciferase reporter analysis was conducted. miR-195-5p overexpression evidently declined the luciferase activity of circ_0020850-WT, while it did not affect the luciferase activity of circ_0020850-MUT (Figure 3B and C). Moreover, miR-195-5p abundance was markedly declined in lung adenocarcinoma tissues compared with normal tissues (Figure 3D). Similarly, miR-195-5p level was evidently reduced in A549 and PC-9 cells in comparison to BEAS-2B cells (Figure 3E). Additionally, miR-195-5p abundance in lung adenocarcinoma tissues was inversely related with circ_0020850 level ( $\mathrm{r}=$ $-0.6361, P<0.0001$ ) (Figure 3F). Besides, the influence of circ_0020850 on miR-195-5p expression was assessed. As displayed in Figure 3G, miR-195-5p abundance was evidently elevated via circ_0020850 silence, which was weakened via transfection of anti-miR-195$5 p$. These data suggested that miR-195-5p was targeted via circ_0020850.

\section{miR-195-5p Knockdown Reverses the Influence of circ_0020850 Silence on Lung Adenocarcinoma Development}

To explore whether miR-195-5p was relevant to circ_0020850-mediated regulation of lung adenocarcinoma development, A549 and PC-9 cells were transfected with si-NC, si-circ_0020850, si-circ_0020850+ anti-miR-NC or anti-miR-195-5p. As displayed in Figure 4A-C, miR-195-5p knockdown abolished the inhibitive effect of circ_0020850 silence on cell proliferation via restoring cell viability and clone ability. Furthermore, miR-195-5p down-regulation weakened the silence of circ_0020850-induced apoptosis in A549 and PC-9 cells (Figure 4D). In addition, miR-195-5p deficiency attenuated knockdown of circ_0020850mediated inhibition of cell migration and invasion (Figure 4E and F). Besides, miR-195-5p knockdown mitigated interference of circ_0020850-mediated regulatory role in the abundances of CyclinD1, Bcl-2, Cleaved-casp-3, Vimentin, N-cadherin and E-cadherin (Figure 4G-J). These results indicated that miR-195-5p knockdown alleviated the inhibitive role of circ_0020850 silence in lung adenocarcinoma development in vitro.

\section{IRS2 is Targeted via miR-195-5p}

To further analyze the regulatory network, the target of miR-195-5p was predicted via starBase. IRS2 was a target and the binding sequence of miR-195-5p and IRS2 is exhibited in Figure 5A. To validate this prediction, the IRS2 $3^{\prime}$ UTR-WT and IRS2 3 'UTR-MUT were constructed. The results of dual-luciferase reporter analysis showed that miR-195-5p overexpression reduced the luciferase activity of IRS2 3'UTR-WT, but it did not alter the luciferase activity of IRS2 3'UTR-MUT (Figure 5B and C). Furthermore, IRS2 abundance was evidently up-regulated in lung adenocarcinoma tissues and cells (Figure 5D-G). Additionally, IRS2 level in lung adenocarcinoma tissues was negatively associated with miR-195-5p expression $(\mathrm{r}=-0.6291, \quad P<0.0001)$ (Figure $5 \mathrm{H}$ ). Besides, the influence of miR-195-5p on IRS2 expression was investigated. As shown in Figure 5I and J, IRS2 level was evidently decreased via miR-195-5p overexpression, which was restored via transfection of pcDNA-IRS2. These results indicated that IRS2 was targeted via miR-195-5p. 
A

\begin{tabular}{|c|c|}
\hline $\begin{array}{l}\text { circ_0020850-WT } \\
\text { miR-195-5p }\end{array}$ & $\begin{array}{r}5^{\prime} \text { UUAAACA-UGC-GUGCUGCUU 3' } \\
\text { I I I I I I I | | | } \\
\text { 3' CGGUUAUAAAGAC ACG ACG AU 5' }\end{array}$ \\
\hline irc & C-CUl \\
\hline
\end{tabular}

B

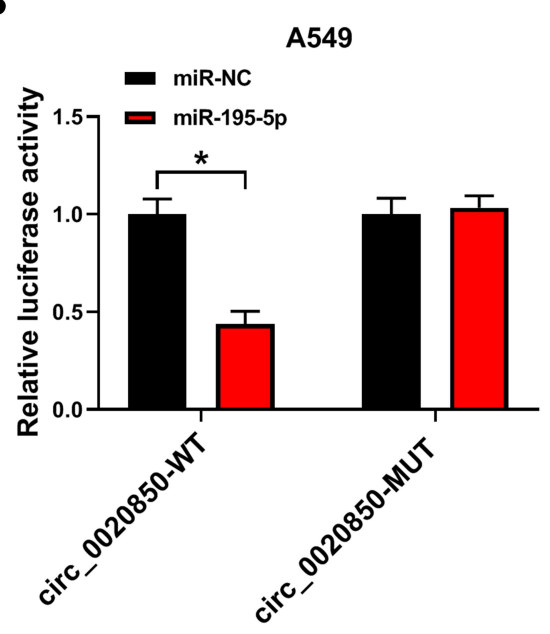

D

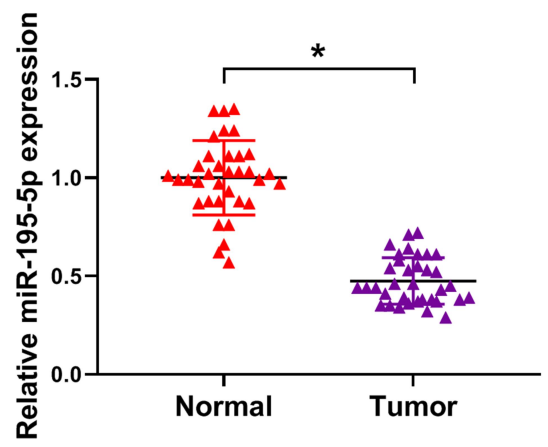

F

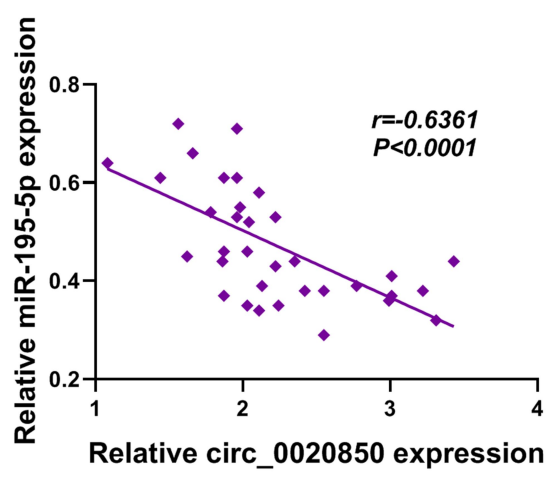

C

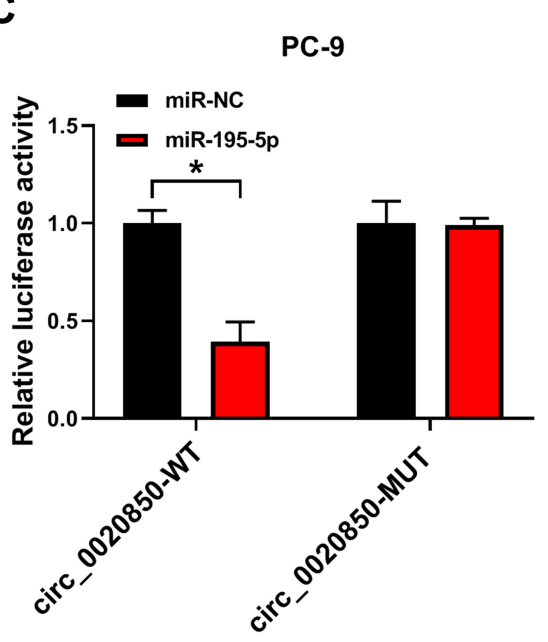

E
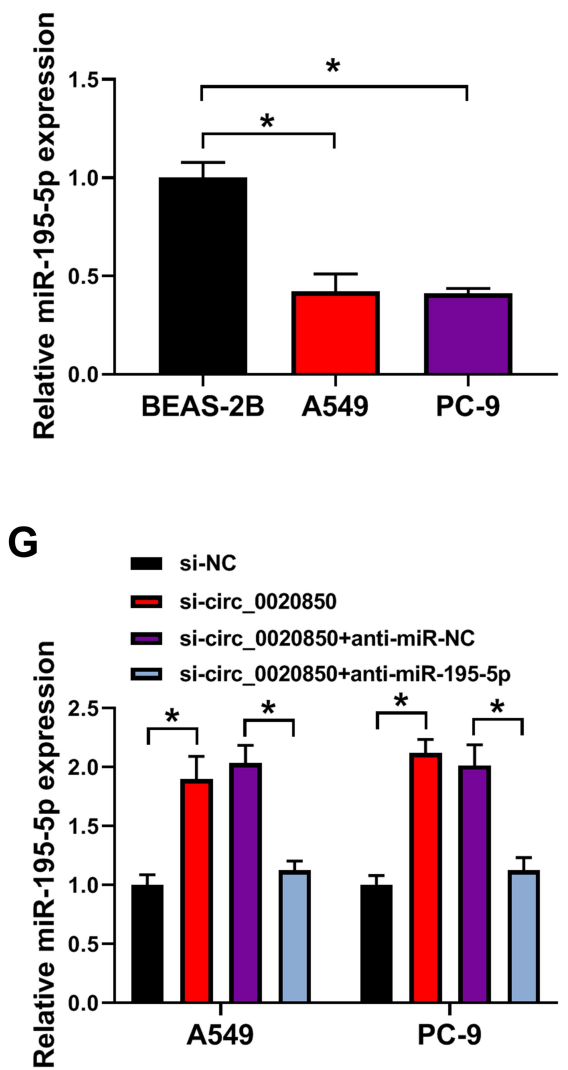

Figure 3 The relationship of circ_0020850 and miR-195-5p. (A) The binding sequence of circ_0020850 and miR-195-5p. (B and C) Luciferase activity was detected in A549 and PC-9 cells with transfection of circ_0020850-WT or circ_0020850-MUT and miR-195-5p mimic or miR-NC. (D and E) miR-195-5p abundance was measured in lung adenocarcinoma tissues and cells. (F) The association between circ 0020850 and miR-195-5p in lung adenocarcinoma tissues. (G) miR-195-5p level was examined in A549 and PC-9 cells with transfection of si-NC, si-circ_0020850, si-circ_0020850 + anti-miR-NC or anti-miR-195-5p. $* P<0.05$. 
A

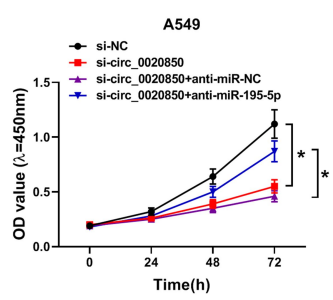

B

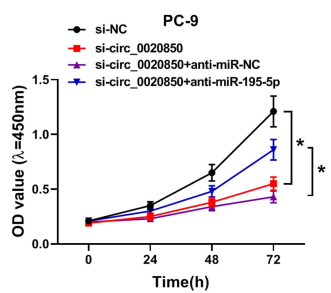

C

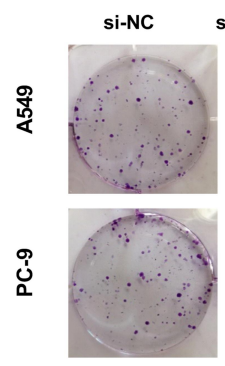

D

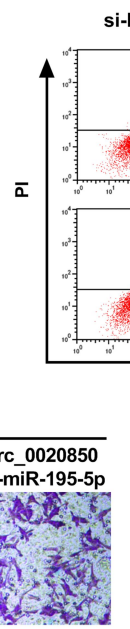

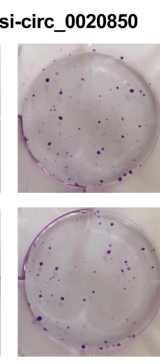

si-circ_0020850
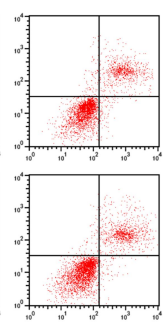

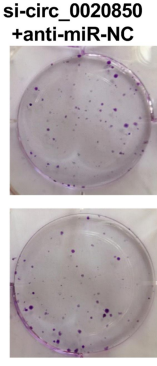

si-circ_0020850
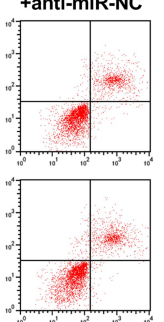

Annexin V-FITC

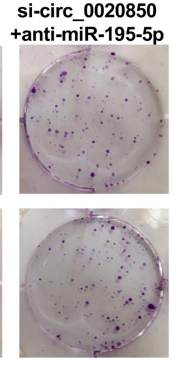

si-circ_0020850
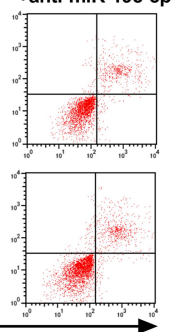
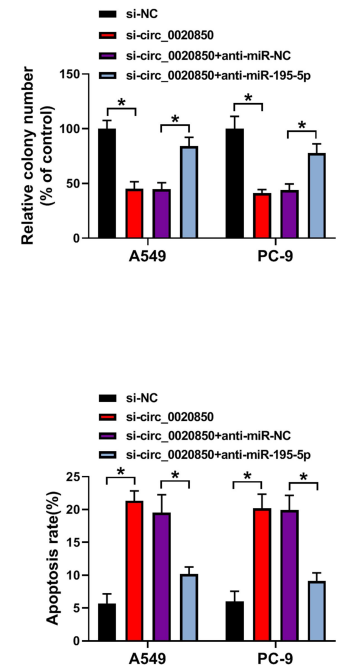

E

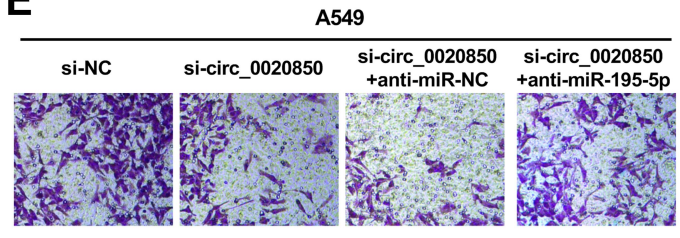

F

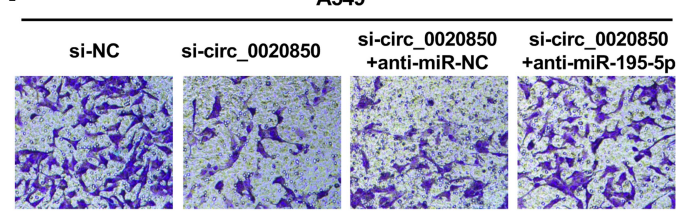

G
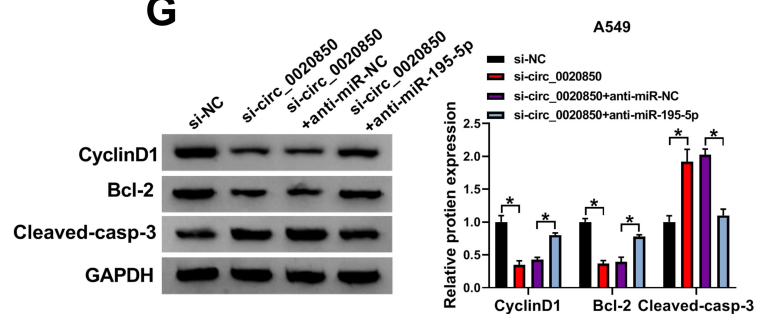

I

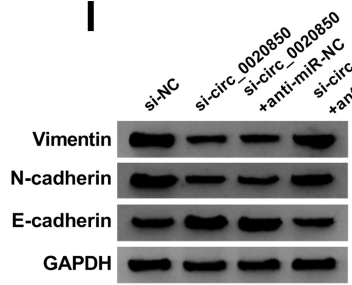

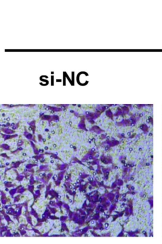

PC-9
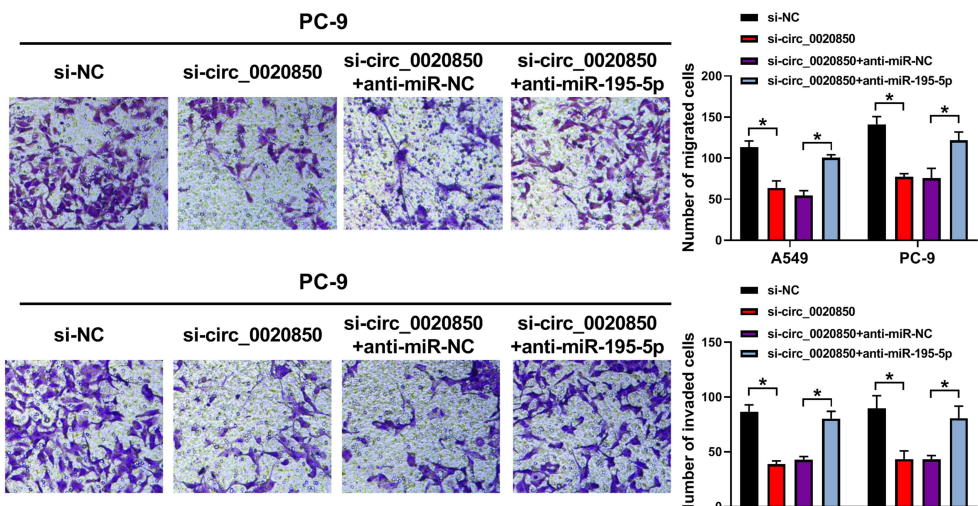

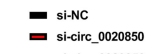

- si-circ 0020850
150 si-circ0020850+anti-miR-NC

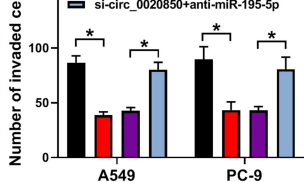

H
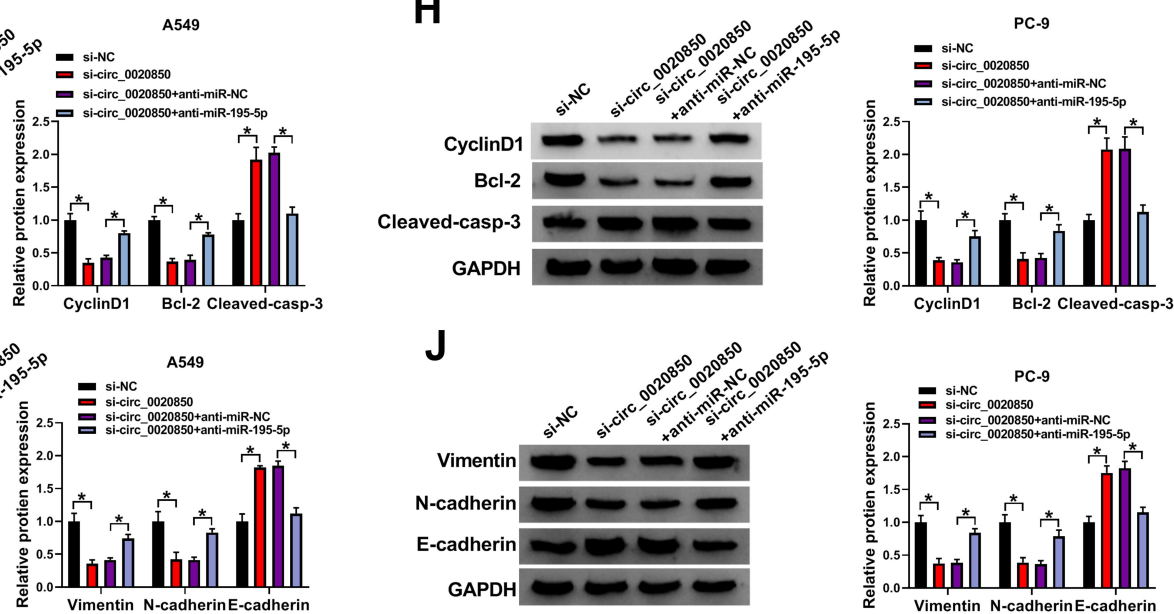

J

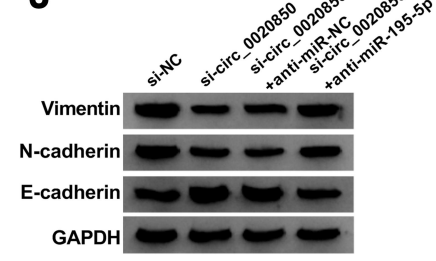

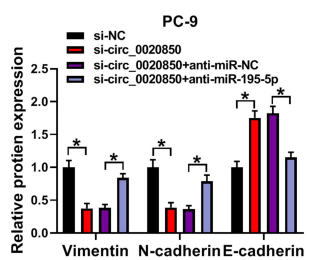

Figure 4 The influence of miR-195-5p on circ 0020850-mediated lung adenocarcinoma development. Cell viability (A and B), colony formation (C), apoptotic rate (D), migration and invasion (E and F), and CyclinDI, Bcl-2, Cleaved-casp-3, Vimentin, N-cadherin and E-cadherin protein levels (G-J) were examined in cells with transfection of si-NC, si-circ_0020850, si-circ_0020850 + anti-miR-NC or anti-miR-195-5p. $* P<0.05$. 
A

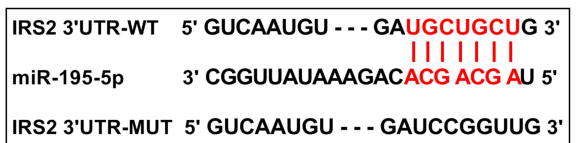

B

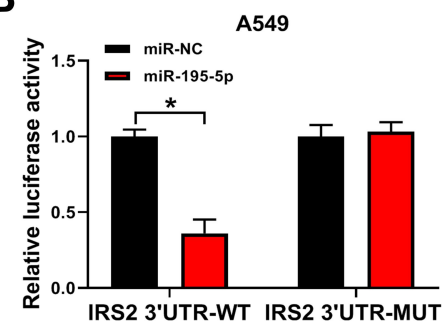

C

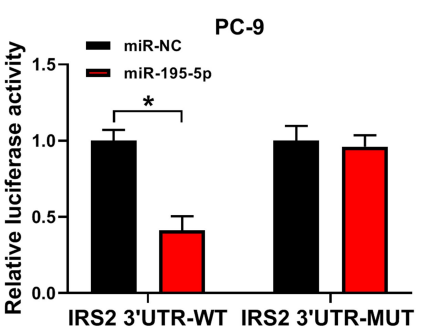

D

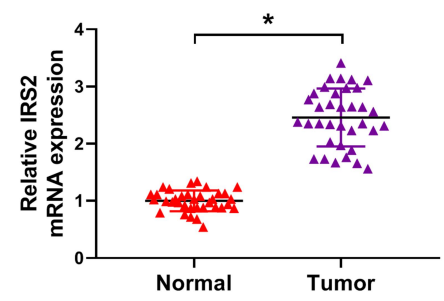

G

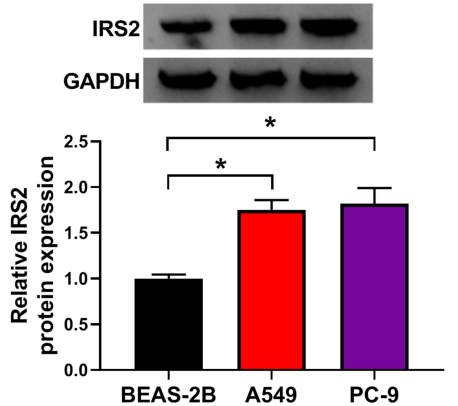

E

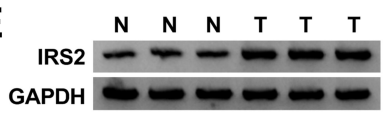

H

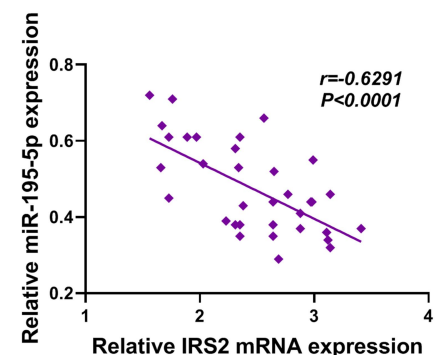

F

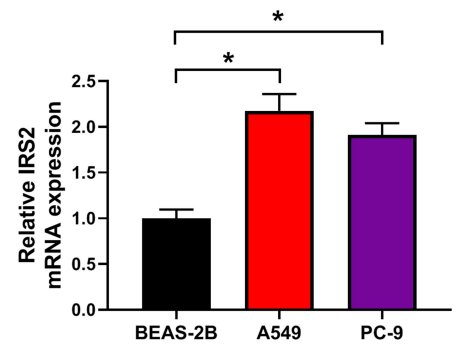

I

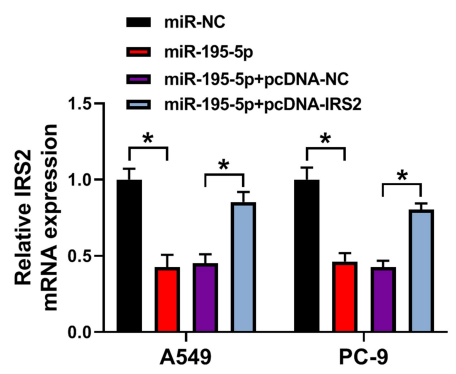

J

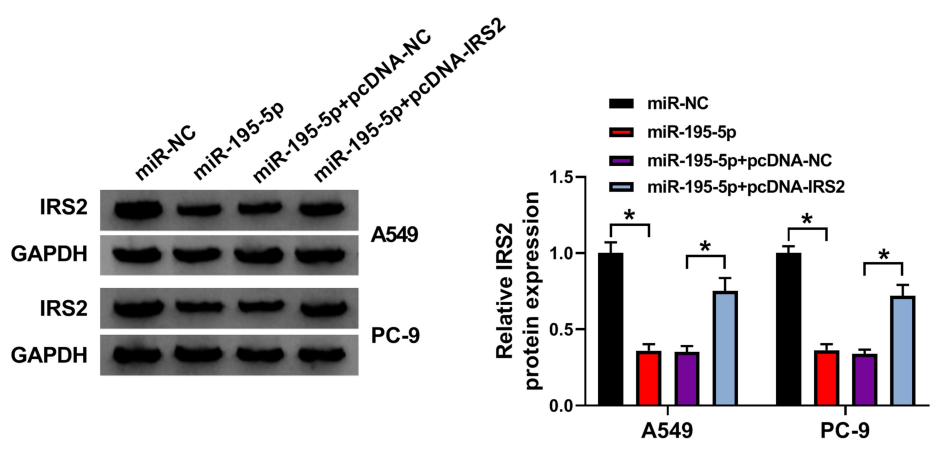

Figure 5 The relationship of miR-195-5p and IRS2. (A) The binding sequence of miR-195-5p and IRS2. (B and C) Luciferase activity was tested in cells with transfection of IRS2 3'UTR-WT or IRS2 3'UTR-MUT and miR-195-5p mimic or miR-NC. (D-G) IRS2 expression was detected in lung adenocarcinoma tissues and cells. (H) The correlation between miR-195-5p and IRS2 in lung adenocarcinoma tissues. (I and J) IRS2 abundance was examined in A549 and PC-9 cells with transfection of miR-NC, miR-195-5p mimic, miR-195-5p mimic + pcDNA-NC or pcDNA-IRS2. $* P<0.05$.

\section{miR-195-5p Inhibits Lung}

\section{Adenocarcinoma Development by Targeting IRS2}

To explore the function of miR-195-5p in lung adenocarcinoma development and whether it required IRS2, A549 and PC-9 cells were transfected with miR-NC, miR-195-5p mimic, miR-195-5p mimic + pcDNA-NC or pcDNA-IRS2. As exhibited in Figure 6A-C, miR-195-5p overexpression significantly repressed cell proliferation by decreasing cell viability and clone ability, which was mitigated via IRS2 overexpression. Moreover, miR-195-5p addition evidently promoted cell apoptosis, which was weakened by IRS2 up- 
A

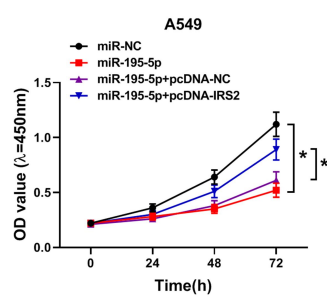

B

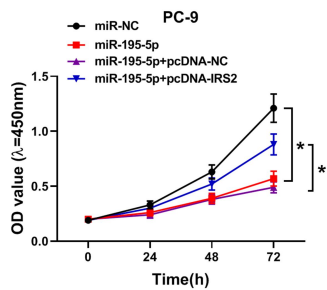

C

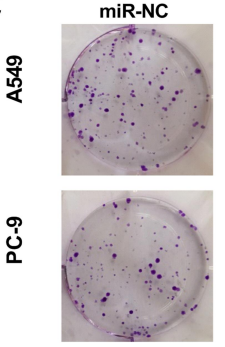

D

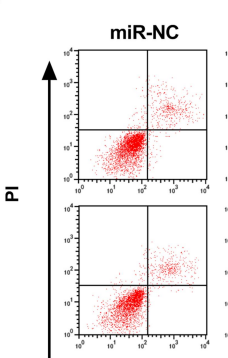

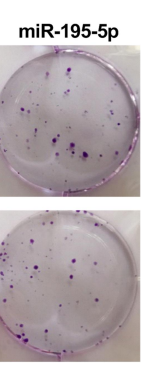

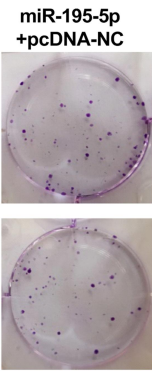

miR-195-5p miR-195-5p
+ pcDNA-NC
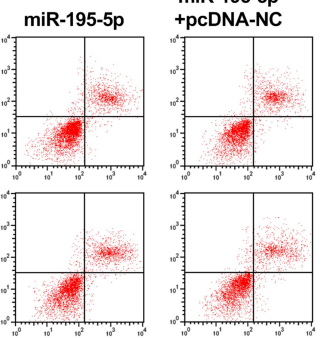

E

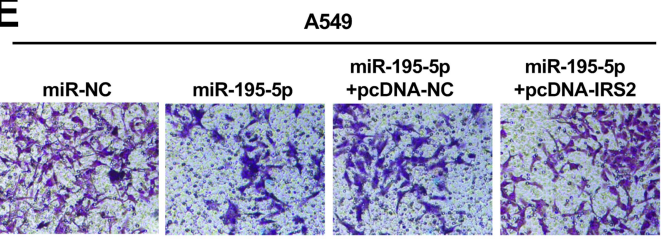

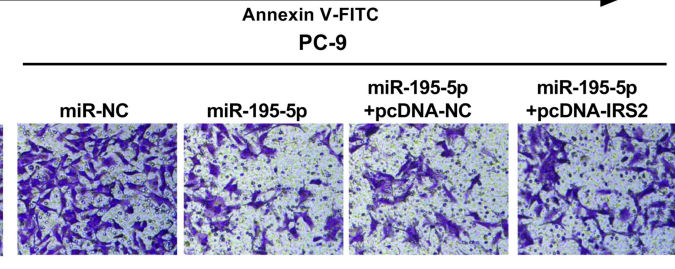

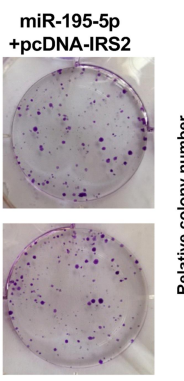

miR-195-5p +pcDNA-IRS2
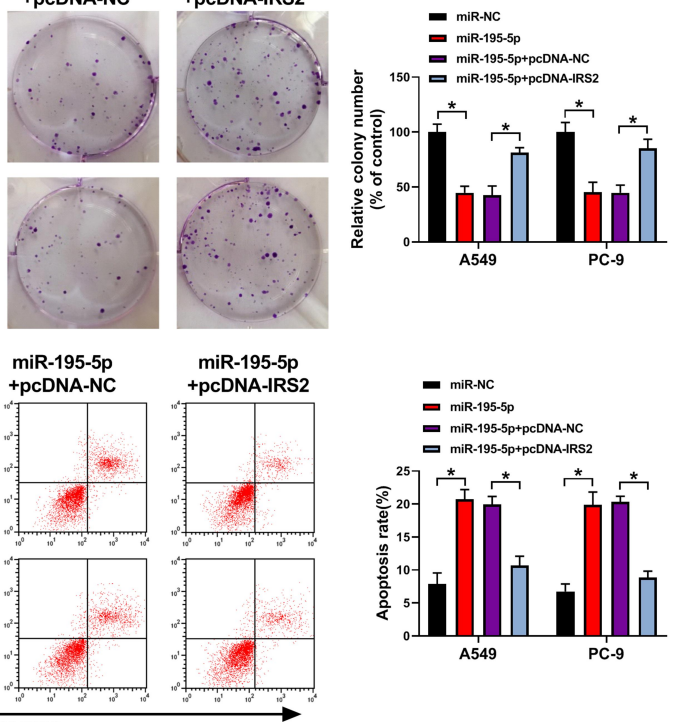

- miR-NC

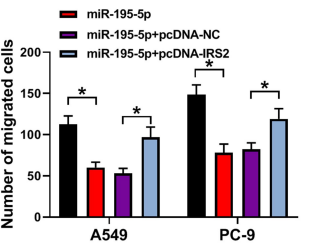

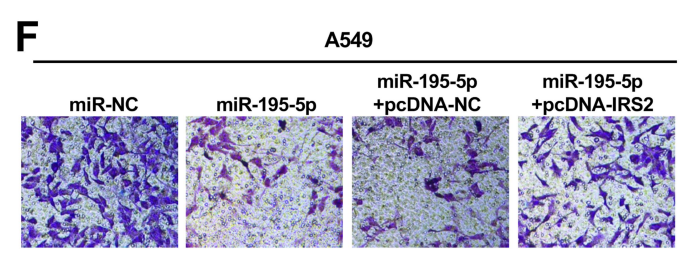
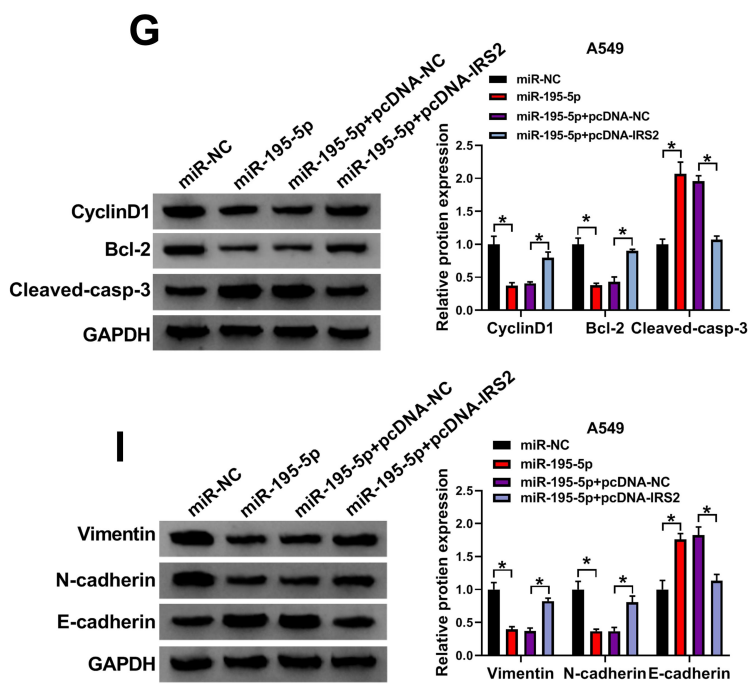
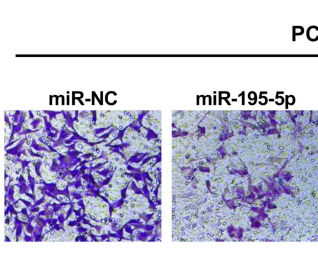

H
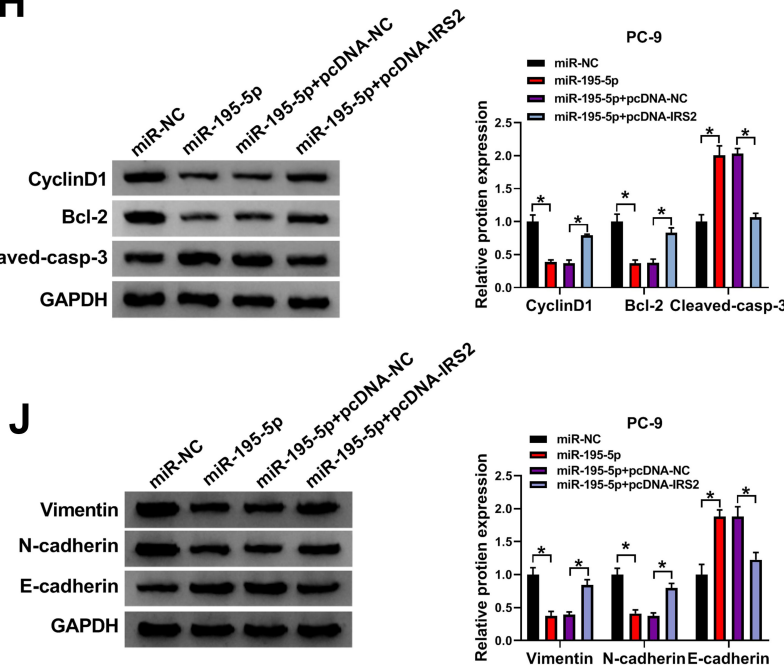

Figure 6 The influence of miR-195-5p and IRS2 on lung adenocarcinoma development. Cell viability (A and B), colony formation (C), apoptotic rate (D), migration and invasion (E and F), and CyclinDI, Bcl-2, Cleaved-casp-3, Vimentin, N-cadherin and E-cadherin protein levels (G-J) were detected in cells with transfection of miR-NC, miR195-5p mimic, miR-195-5p mimic + pcDNA-NC or pcDNA-IRS2. $* p<0.05$. 


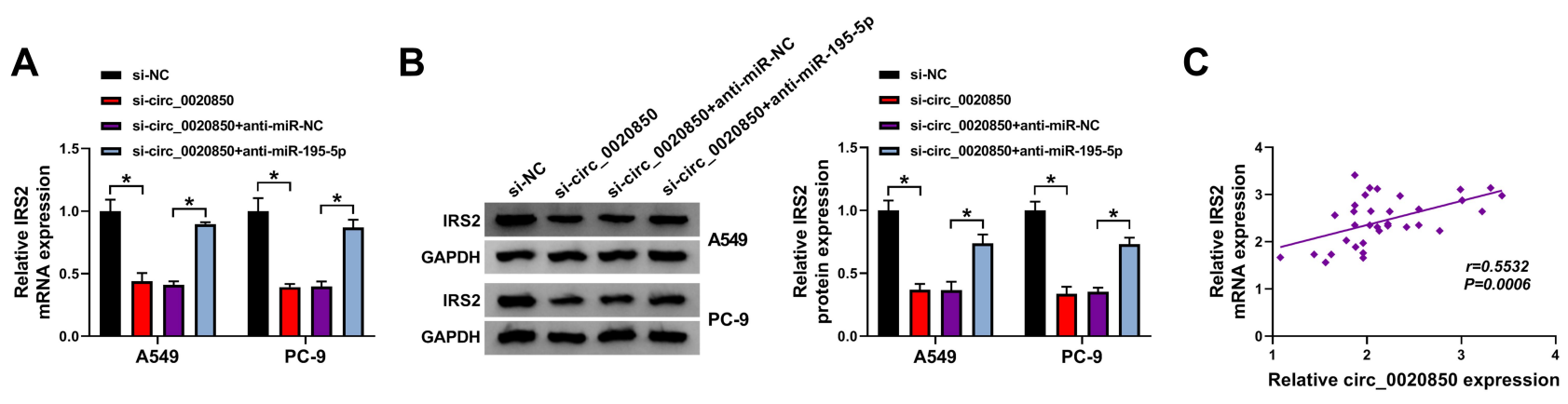

Figure 7 The influence of circ_0020850 and miR-195-5p on IRS2 expression in lung adenocarcinoma. (A and B) IRS2 expression was detected in A549 and PC-9 cells with transfection of si-NC, si-circ_0020850, si-circ_0020850 + anti-miR-NC or anti-miR-195-5p. (C) The correlation between circ_0020850 and IRS2 in lung adenocarcinoma tissues. $* P<0.05$.

regulation (Figure 6D). Additionally, miR-195-5p overexpression markedly repressed cell migration and invasion, which was attenuated via IRS2 restoration (Figure 6E and F). Besides, miR-195-5p overexpression obviously declined CyclinD1, Bcl-2, Vimentin and N-cadherin expression and enhanced Cleaved-casp-3 and E-cadherin levels, which was reversed via IRS2 overexpression (Figure 6G-J). These data suggested that miR-195-5p inhibited lung adenocarcinoma development in vitro via IRS2.

\section{circ_0020850 Regulates IRS2 via miR- $195-5 p$}

To explore how circ_0020850 regulated IRS2, the influence of circ_0020850 on IRS2 expression was assessed in A549 and PC-9 cells. As displayed in Figure 7A and B, IRS2 expression was evidently reduced via circ_0020850 silence, which was restored via miR-195-5p knockdown. Furthermore, IRS2 abundance in lung adenocarcinoma tissues was positively correlated with circ_0020850 level $(\mathrm{r}=0.5532, P=0.0006)$ (Figure $7 \mathrm{C})$. These results indicated that circ_0020850 could target IRS2 by competitively binding with miR-195-5p.

\section{circ_0020850 Knockdown Reduces Xenograft Tumor Growth}

To explore the function of circ_0020850 in lung adenocarcinoma in vivo, PC-9 cells stably transfected with shcirc_0020850 or sh-NC were used to establish xenograft model, and the mice were divided into sh-circ_0020850 or sh-NC group. After 27 days, tumor volume and weight were evidently declined in sh-circ_0020850 group compared with sh-NC group (Figure 8A and B). Next, circ_0020850, miR-195-5p and IRS2 levels were examined in the tumor tissues. As displayed in Figure 8C-F, circ_0020850 and IRS2 abundances were markedly reduced and miR-195-5p was elevated in shcirc_0020850 group in comparison to sh-NC group. These data indicated that circ_0020850 silence decreased lung adenocarcinoma xenograft tumor growth.

\section{Discussion}

Lung adenocarcinoma is a major subtype of lung cancer. ${ }^{18}$ The dysregulated circRNAs are associated with lung adenocarcinoma development. ${ }^{19}$ In this research, we aimed to investigate how and whether circ_0020850 regulated lung adenocarcinoma development. Here we found that circ_0020850 interference could inhibit lung adenocarcinoma progression, and we were the first to indicate it was associated with miR-195-5p/IRS2 axis.

Basing on the GSE101684 microarray data, ${ }^{7}$ we chose 10 up-regulated and 10 down-regulated circRNAs and found that circ_0020850 expression was increased most. Moreover, our study showed that circ_0020850 level was enhanced in lung adenocarcinoma tissues and cells, implying the increased circ_0020850 might be involved in lung adenocarcinoma malignancy. To explore the function of circ_0020850, we performed the loss-of-function experiments and found that circ_0020850 down-regulation suppressed cell proliferation, migration and invasion and contributed to apoptosis. This indicated circ_0020850 inhibition played an anti-cancer role in lung adenocarcinoma.

The competing endogenous RNA network mediated via circRNAs reveals the potential mechanism in lung cancer. ${ }^{20}$ We first explored the interaction of circRNA/ miRNA and identified miR-195-5p was targeted via circ_0020850. Zhou et al suggested that miR-195-5p could restrain proliferation, migration and invasion in lung adenocarcinoma by decreasing Apelin. ${ }^{11}$ Additionally, miR-195-5p could inhibit cell proliferation 
A

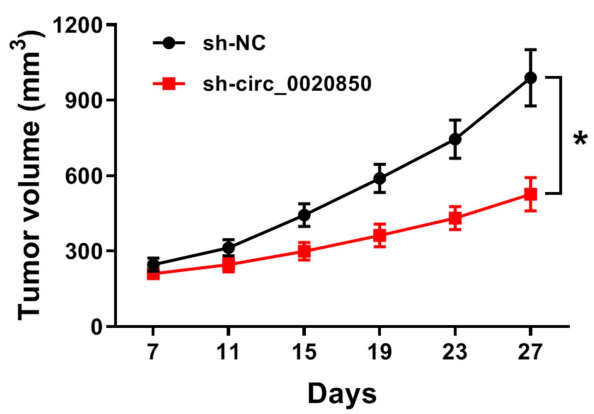

C

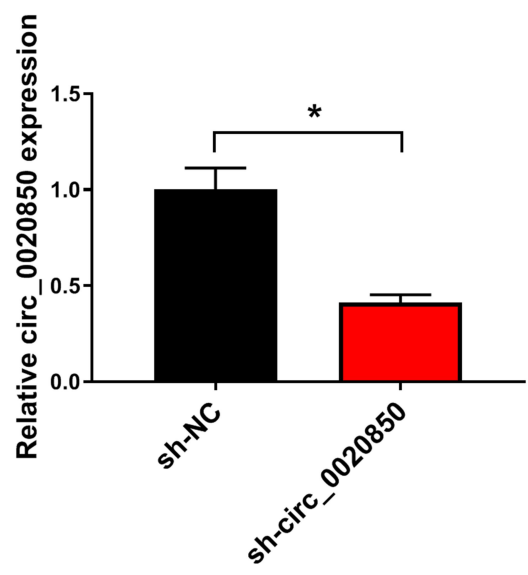

E

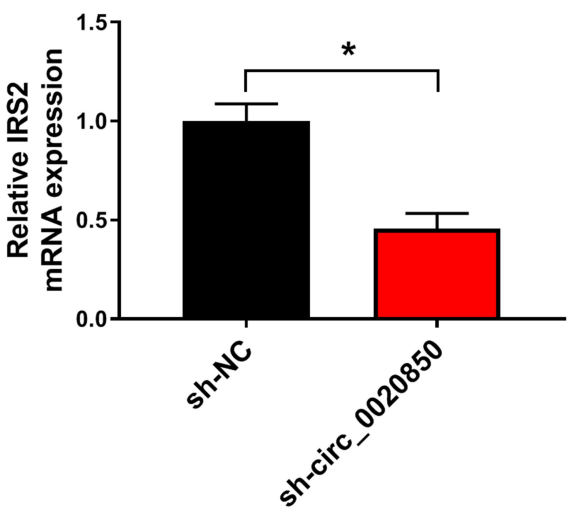

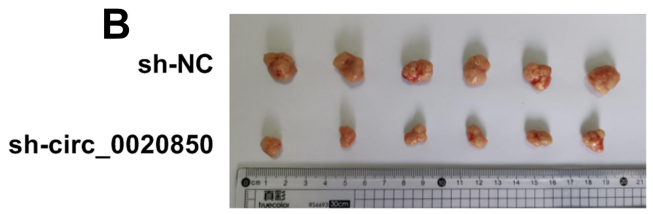

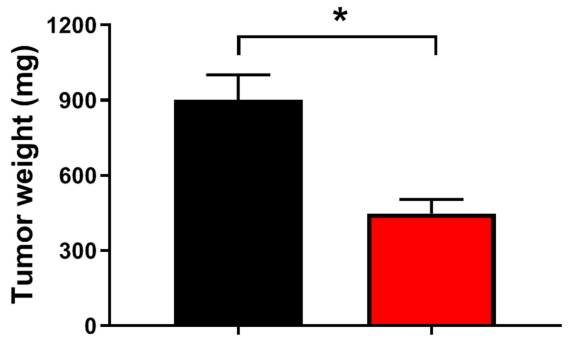

$s^{c}$

D
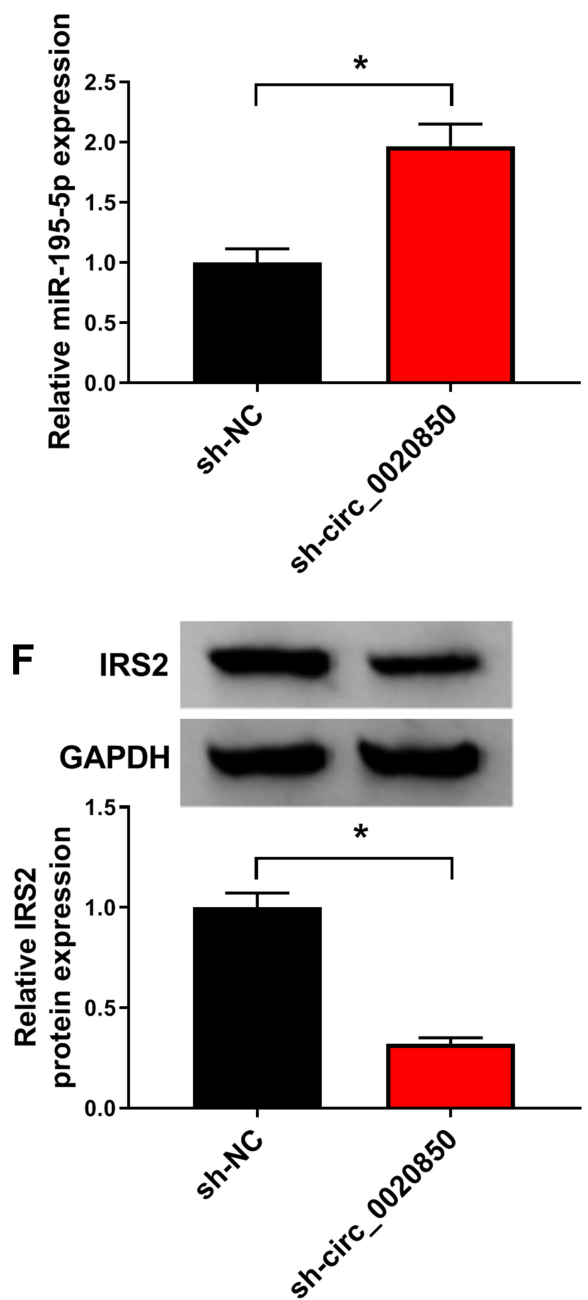

Figure 8 The influence of circ_0020850 on lung adenocarcinoma growth in xenograft model. (A) Tumor volume was detected every 4 days. (B) Tumor weight was measured at the end point. (C-F) circ_0020850, miR-195-5P and IRS2 abundances were examined in tumor tissues of each group. ${ }^{*} P<0.05$. 
and promote apoptosis via regulating cyclin E1 and centrosomal protein $55 .^{21,22}$ These reports suggested the tumor-suppressive role of miR-195-5p in lung adenocarcinoma. Similarly, here we also found that miR-195-5p overexpression repressed cell proliferation, migration and invasion and promoted apoptosis. Furthermore, miR-1955 p knockdown weakened the influence of circ_0020850 silence on lung adenocarcinoma development, indicating that circ_0020850 could regulate lung adenocarcinoma via sponging miR-195-5p.

Next, we explored the interaction of miRNA/mRNA mediated via miR-195-5p and confirmed IRS2 was targeted via miR-195-5p. Gao et al reported that IRS2 abundance was enhanced in lung cancer, and IRS2 silence inhibited cell proliferation and metastasis. ${ }^{23}$ Tan et al suggested IRS2 overexpression reversed miR-200a-3p-mediated inhibition of cell proliferation, migration and invasion in lung cancer. ${ }^{15}$ Furthermore, increasing evidences suggested IRS2 could be targeted via miR-338-3p to contribute to lung cancer cell proliferation, migration and invasion. ${ }^{14,24,25}$ These reports proposed the oncogenic function of IRS2 in lung cancer. Similarly, our work also validated the promoting role of IRS2 in lung adenocarcinoma development, revealed via which IRS2 abated the anti-cancer role of miR-195-5p. Besides, we explored how circ_0020850 regulated IRS2 and found that circ_0020850 could modulate IRS2 expression via competitively binding with miR-195-5p. Thus, we concluded that circ_0020850 targeted IRS2 via miR-195-5p to participate in lung adenocarcinoma development in vitro. The animal model attempts to capture the complex microenvironment in human cancers. ${ }^{26}$ In this work, we established murine xenograft model to assess the function of circ_0020850 on lung adenocarcinoma tumorigenesis in vivo. Here we found that circ_0020850 knockdown attenuated lung adenocarcinoma growth.

However, there were some limitations in the current work. Firstly, the clinical value of circ_0020850 was not analyzed due to the smaller sample size. Secondly, the drug sensitivity was also important for cancer treatment, while the current work did not perform the drug sensitivity assay, which would be explored in further study. Finally, the potential signaling pathway addressed via circ_0020850/miR-195-5p/IRS2 axis was absent. Previous studies reported that protein kinase B (Akt) and extracellular signal-related kinases (ERK) pathways controlled fundamental physiological processes, like proliferation, metabolism, migration, invasion and cell death in lung cancer. ${ }^{27,28}$ Moreover, IRS2 could activate this signaling to regulate cell proliferation, migration, invasion and apoptosis in multiple conditions, including lung cancer. $^{29,30}$ Hence, we hypothesized that circ_0020850 might activate IRS2-mediated Akt and ERK pathways to promote lung adenocarcinoma development, which should be analyzed in future.

In conclusion, circ_0020850 silence repressed lung adenocarcinoma development, possibly via modulating miR-195-5p/IRS2 axis. This research indicates a new target for the treatment of lung adenocarcinoma.

\section{Abbreviations}

circRNAs, circular RNAs; circ_0020850, hsa_circ_0020850; miR-195-5p, microRNA-195-5p; IRS2, insulin receptor substrate 2 .

\section{Funding}

There is no funding to report.

\section{Disclosure of Interest}

The authors declare that they have no financial conflicts of interest. The authors report no conflicts of interest for this work.

\section{References}

1. Testa U, Castelli G, Pelosi E. Lung cancers: molecular characterization, clonal heterogeneity and evolution, and cancer stem cells. Cancers (Basel). 2018;10(8):248. doi:10.3390/cancers10080248

2. Denisenko TV, Budkevich IN, Zhivotovsky B. Cell death-based treatment of lung adenocarcinoma. Cell Death Dis. 2018;9(2):117. doi:10.1038/s41419-017-0063-y

3. Kristensen LS, Andersen MS, Stagsted LVW, Ebbesen KK, Hansen TB, Kjems J. The biogenesis, biology and characterization of circular RNAs. Nat Rev Genet. 2019;20(11):675-691. doi:10.1038/s41576019-0158-7

4. Wang C, Tan S, Liu WR, et al. RNA-Seq profiling of circular RNA in human lung adenocarcinoma and squamous cell carcinoma. Mol Cancer. 2019;18(1):134. doi:10.1186/s12943-019-1061-8

5. Yao Y, Hua Q, Zhou Y, Shen H. CircRNA has_circ_0001946 promotes cell growth in lung adenocarcinoma by regulating miR-135a-5p/SIRT1 axis and activating Wnt/beta-catenin signaling pathway. Biomed Pharmacother. 2019;111:1367-1375. doi:10.1016/j. biopha.2018.12.120

6. Gao N, Ye B. Circ-SOX4 drives the tumorigenesis and development of lung adenocarcinoma via sponging miR-1270 and modulating PLAGL2 to activate WNT signaling pathway. Cancer Cell Int. 2020;20:2. doi:10.1186/s12935-019-1065-x

7. Zhao J, Li L, Wang Q, Han H, Zhan Q, Xu M. CircRNA expression profile in early-stage lung adenocarcinoma patients. Cell Physiol Biochem. 2017;44(6):2138-2146. doi:10.1159/000485953

8. Inamura K, Ishikawa Y. MicroRNA in lung cancer: novel biomarkers and potential tools for treatment. J Clin Med. 2016;5(3):36. doi: $10.3390 /$ jcm5030036

9. Arima C, Kajino T, Tamada Y, et al. Lung adenocarcinoma subtypes definable by lung development-related miRNA expression profiles in association with clinicopathologic features. Carcinogenesis. 2014;35 (10):2224-2231. doi:10.1093/carcin/bgu127 
10. Li L, Feng T, Zhang W, et al. MicroRNA biomarker hsa-miR-195-5p for detecting the risk of lung cancer. Int $J$ Genomics. 2020;2020:7415909. doi:10.1155/2020/7415909

11. Zhou Y, Zhao M, Du Y, et al. MicroRNA-195 suppresses the progression of lung adenocarcinoma by directly targeting apelin. Thorac Cancer. 2019;10(6):1419-1430. doi:10.1111/1759-7714.13087

12. Yuan C, Xiang L, Bai R, et al. MiR-195 restrains lung adenocarcinoma by regulating $\mathrm{CD} 4+\mathrm{T}$ cell activation via the $\mathrm{CCDC} 88 \mathrm{C} / \mathrm{Wnt}$ signaling pathway: a study based on the Cancer Genome Atlas (TCGA), Gene Expression Omnibus (GEO) and bioinformatic analysis. Ann Transl Med. 2019;7(12):263. doi:10.21037/atm.2019.05.54

13. Piper AJ, Clark JL, Mercado-Matos J, et al. Insulin receptor substrate-1 (IRS-1) and IRS-2 expression levels are associated with prognosis in non-small cell lung cancer (NSCLC). PLoS One. 2019;14(8):e0220567. doi:10.1371/journal.pone.0220567

14. Zhang P, Shao G, Lin X, Liu Y, Yang Z. MiR-338-3p inhibits the growth and invasion of non-small cell lung cancer cells by targeting IRS2. Am J Cancer Res. 2017;7(1):53-63.

15. Tan T, Xu XH, Lu XH, Wang XW. MiRNA-200a-3p suppresses the proliferation, migration and invasion of non-small cell lung cancer through targeting IRS2. Eur Rev Med Pharmacol Sci. 2020;24 (2):712-720. doi:10.26355/eurrev_202001_20050

16. Chomczynski P, Sacchi N. The single-step method of RNA isolation by acid guanidinium thiocyanate-phenol-chloroform extraction: twenty-something years on. Nat Protoc. 2006;1(2):581-585. doi:10.1038/nprot.2006.83

17. Livak KJ, Schmittgen TD. Analysis of relative gene expression data using real-time quantitative PCR and the 2(-Delta Delta C(T)) Method. Methods. 2001;25(4):402-408. doi:10.1006/meth.2001.1262

18. Travis WD. Lung cancer pathology: current concepts. Clin Chest Med. 2020;41(1):67-85. doi:10.1016/j.ccm.2019.11.001

19. Zhang $\mathrm{F}$, Zhao X, Dong $\mathrm{H}$, Xu J. circRNA expression analysis in lung adenocarcinoma: comparison of paired fresh frozen and formalin-fixed paraffin-embedded specimens. Biochem Biophys Res Commun. 2018;500(3):738-743. doi:10.1016/j.bbrc.2018.04.145

20. Jin X, Guan Y, Sheng H, Liu Y. Crosstalk in competing endogenous RNA network reveals the complex molecular mechanism underlying lung cancer. Oncotarget. 2017;8(53):91270-91280. doi:10.18632/ oncotarget.20441
21. Liang $\mathrm{Y}$, Rong X, Luo $\mathrm{Y}$, et al. A novel long non-coding RNA LINC00355 promotes proliferation of lung adenocarcinoma cells by down-regulating miR-195 and up-regulating the expression of CCNE1. Cell Signal. 2020;66:109462. doi:10.1016/j. cellsig.2019.109462

22. Luo J, Pan J, Jin Y, Li M, Chen M. MiR-195-5p inhibits proliferation and induces apoptosis of non-small cell lung cancer cells by targeting CEP55. Onco Targets Ther. 2019;12:11465-11474. doi:10.2147/OTT. $\mathrm{S} 226921$

23. Gao J, Feng X, Wang F, et al. microRNA-448 inhibits the progression of non-small-cell lung cancer through regulating IRS2. J Cell Biochem. 2019;120(8):13453-13463. doi:10.1002/jcb.28619

24. Li S, Niu X, Li H, Liang Y, Sun Z, Yan Y. Circ_0000003 promotes the proliferation and metastasis of non-small cell lung cancer cells via miR-338-3p/insulin receptor substrate 2. Cell Cycle. 2019;18 (24):3525-3539. doi:10.1080/15384101.2019.1690883

25. Yang Z, Lin X, Zhang $P$, et al. Long non-coding RNA LINC00525 promotes the non-small cell lung cancer progression by targeting miR-338-3p/IRS2 axis. Biomed Pharmacother. 2020;124:109858. doi:10.1016/j.biopha.2020.109858

26. Langdon SP. Animal modeling of cancer pathology and studying tumor response to therapy. Curr Drug Targets. 2012;13(12):15351547. doi:10.2174/138945012803530152

27. Ciuffreda L, Incani UC, Steelman LS, et al. Signaling intermediates (MAPK and PI3K) as therapeutic targets in NSCLC. Curr Pharm Des. 2014;20(24):3944-3957. doi:10.2174/13816128113196660763

28. Sun Z, Wang Z, Liu X, Wang D. New development of inhibitors targeting the PI3K/AKT/mTOR pathway in personalized treatment of non-small-cell lung cancer. Anticancer Drugs. 2015;26(1):1-14. doi:10.1097/CAD.0000000000000172

29. Martínez-Hervás S, Vinué A, Núñez L, et al. Insulin resistance aggravates atherosclerosis by reducing vascular smooth muscle cell survival and increasing CX3CL1/CX3CR1 axis. Cardiovasc Res. 2014;103(2):324-336. doi:10.1093/cvr/cvu115

30. Cui A, Hua H, Shao T, et al. Aflatoxin B1 induces Src phosphorylation and stimulates lung cancer cell migration. Tumour Biol. 2015;36 (8):6507-6513. doi:10.1007/s13277-015-3341-2

\section{Publish your work in this journal}

Cancer Management and Research is an international, peer-reviewed open access journal focusing on cancer research and the optimal use of preventative and integrated treatment interventions to achieve improved outcomes, enhanced survival and quality of life for the cancer patient.
The manuscript management system is completely online and includes a very quick and fair peer-review system, which is all easy to use. Visit http://www.dovepress.com/testimonials.php to read real quotes from published authors. 